\title{
Existence and nonexistence of positive solutions for fractional-order two-point boundary value problems
}

\author{
Yongping Sun ${ }^{*}$ and Xiaoping Zhang
}

\section{"Correspondence:}

sunyongping@126.com College of Electron and Information, Zhejiang University of Media and Communications, Hangzhou, Zhejiang 310018, China

\begin{abstract}
The purpose of this paper is to establish some results on the existence and nonexistence of positive solutions for a type of nonlinear fractional-order two-point boundary value problems. The main tool is a fixed point theorem of the cone expansion and compression of functional type due to Avery et al. Some examples are presented to illustrate the availability of the main results.

MSC: 34A08; 34B10; 34B15; 34B18
\end{abstract}

Keywords: positive solution; existence and nonexistence; fractional differential equations; boundary value problems; fixed point theorem

\section{Introduction}

This paper investigates the fractional boundary value problem (FBVP for short):

$$
\left\{\begin{array}{l}
{ }^{c} D_{0^{+}}^{\alpha} u(t)+f(t, u(t))=0, \quad t \in(0,1), \\
u(0)=u^{\prime}(0)=u^{\prime \prime \prime}(0)=\cdots=u^{(n-1)}(0)=0, \quad u^{\prime \prime}(1)=0,
\end{array}\right.
$$

where ${ }^{c} D_{0^{+}}^{\alpha}$ is Caputo's fractional derivative. Throughout this paper, we assume that $n \geq 4$ is a fixed integer, $\alpha \in(n-1, n]$, and $f:[0,1] \times[0, \infty) \rightarrow[0, \infty)$ is continuous.

Fractional differential equations can be extensively applied to various disciplines such as physics, mechanics, chemistry, engineering, and many other branches of science. Recently, there have been some papers dealing with the existence and multiplicity of solutions (or positive solutions) of nonlinear fractional differential equations with various boundary conditions (see [1-33] and the references therein). For example, Agarwal et al. [1] and Tian and Liu [2] investigated the singular fractional boundary value problem of the form:

$$
\left\{\begin{array}{l}
{ }^{c} D_{0^{+}}^{\alpha} u(t)+\lambda f(t, u(t))=0, \quad t \in(0,1), \\
u(0)=u^{\prime}(0)=u^{\prime \prime \prime}(0)=\cdots=u^{(n-1)}(0)=0, \quad u^{\prime \prime}(1)=0
\end{array}\right.
$$

where $\alpha \in(n-1, n]$ and $n \geq 4$ is an integer, ${ }^{c} D_{0^{+}}^{\alpha}$ is Caputo's fractional derivative, $f:(0,1) \times$ $(0, \infty) \rightarrow[0, \infty)$ is continuous, that is, $f(t, u)$ may be singular at $t=0,1$ and $u=0$. By constructing a special cone and using an approximation method and fixed point index theory, the authors obtained some results on the existence or nonexistence of one or more positive solutions.

O2014 Sun and Zhang; licensee Springer. This is an Open Access article distributed under the terms of the Creative Commons Attribution License (http://creativecommons.org/licenses/by/2.0), which permits unrestricted use, distribution, and reproduction in any medium, provided the original work is properly cited. 
Fixed point theorems have been applied to various boundary value problems to show the existence and multiplicity of positive solutions in the last two decades. Recently, Avery et al. [34] generalized the fixed point theorem of a cone expansion and compression of norm type by replacing the norms with two functionals satisfying certain conditions to produce a fixed point theorem of the cone expansion and compression of functional type, and then they applied the fixed point theorem to verify the existence of a positive solution to a second order conjugate boundary value problem.

Motivated greatly by the above-mentioned work, by constructing a special cone and using the fixed point theorem of cone expansion and compression of functional type due to Avery et al., in this paper, we obtain some sufficient conditions for the existence of positive solutions for FBVP (1.1). We also discuss the nonexistence of positive solutions. Here, as usual, by a positive solution to FBVP (1.1), we mean a solution $u(t)$ such that $u(t)>0$ on $(0,1]$. To the author's best knowledge, no paper in the existing literature can be found using this fixed point theorem to prove the existence of a positive solution to the boundary value problem of nonlinear fractional-order differential equations.

We organize the rest of this paper as follows. In Section 2, we present some definitions and background results. We also state a fixed point theorem of cone expansion and compression of functional type due to Avery et al. The expression and properties of Green's function will be given in Section 3. The existence and nonexistence results are proved in Section 4. We end the paper with four examples of applications in Section 5.

\section{Preliminaries}

To make this paper self-contained, in this section, we recall some definitions and properties of the fractional calculus. We also state a fixed point theorem of a cone expansion and compression of functional type due to Avery et al. The presentation here and more information on fractional calculus can be found in, for example, [35, 36].

Definition 2.1 Let $q \geq 0, n=[q]+1$. If $h \in A C^{n}[0,1]$, then Caputo's derivative of the fractional order $q$ is defined by

$$
{ }^{c} D_{0^{+}}^{q} h(t)=\frac{1}{\Gamma(n-q)} \int_{0}^{t}(t-s)^{n-q-1} h^{(n)}(s) d s .
$$

Here $\Gamma$ is the usual Gamma function given by $\Gamma(q)=\int_{0}^{\infty} e^{-t} t^{q-1} d t, \alpha>0$.

Definition 2.2 If $h \in C[0,1]$ and $q>0$, then the Riemann-Liouville fractional integral of order $q$ is defined by

$$
I_{0+}^{q} h(t)=\frac{1}{\Gamma(q)} \int_{0}^{t}(t-s)^{q-1} h(s) d s, \quad t>0 .
$$

The following definitions can be found in [37].

Definition 2.3 Let $E$ be a real Banach space. A nonempty closed convex set $P \subset E$ is called a cone of $E$ if it satisfies the following two conditions:

(1) $u \in P, \lambda>0$ implies $\lambda u \in P$;

(2) $u \in P,-u \in P$ implies $u=0$. 
Every cone $P \subset E$ induces an ordering in $E$ given by $u \leq v$ if and only if $v-u \in P$.

Definition 2.4 Let $E$ be a real Banach space. An operator $T: E \rightarrow E$ is said to be completely continuous if it is continuous and maps bounded sets into precompact sets.

Definition 2.5 A map $\alpha$ is said to be a nonnegative continuous concave functional on a cone $P$ of a real Banach space $E$ if $\alpha: P \rightarrow[0,+\infty)$ is continuous and

$$
\alpha(\lambda u+(1-\lambda) v) \geq \lambda \alpha(u)+(1-\lambda) \alpha(v), \quad u, v \in P, 0 \leq \lambda \leq 1 .
$$

Similarly we said the map $\beta$ is a nonnegative continuous convex functional on a cone $P$ of a real Banach space $E$ if $\beta: P \rightarrow[0,+\infty)$ is continuous and

$$
\beta(\lambda u+(1-\lambda) v) \leq \lambda \beta(u)+(1-\lambda) \beta(v), \quad u, v \in P, 0 \leq \lambda \leq 1 .
$$

We say the map $\gamma$ is sublinear functional if

$$
\gamma(\lambda u) \leq \lambda \gamma(u), \quad u \in P, 0 \leq \lambda \leq 1 .
$$

Property A1 Let $P$ be a cone in a real Banach space $E$ and $\Omega$ be a bounded open subset of $E$ with $0 \in \Omega$. Then a continuous functional $\beta: P \rightarrow[0, \infty)$ is said to satisfy Property A1 if one of the following conditions holds:

(a) $\beta$ is convex, $\beta(0)=0, \beta(u) \neq 0$ if $u \neq 0$, and $\inf _{u \in P \cap \partial \Omega} \beta(u)>0$;

(b) $\beta$ is sublinear, $\beta(0)=0, \beta(u) \neq 0$ if $u \neq 0$, and $\inf _{u \in P \cap \partial \Omega} \beta(u)>0$;

(c) $\beta$ is concave and unbounded.

Property A2 Let $P$ be a cone in a real Banach space $E$ and $\Omega$ be a bounded open subset of $E$ with $0 \in \Omega$. Then a continuous functional $\beta: P \rightarrow[0, \infty)$ is said to satisfy Property A2 if one of the following conditions holds:

(a) $\beta$ is convex, $\beta(0)=0, \beta(u) \neq 0$ if $u \neq 0$;

(b) $\beta$ is sublinear, $\beta(0)=0, \beta(u) \neq 0$ if $u \neq 0$;

(c) $\beta(u+v) \geq \beta(u)+\beta(v)$ for all $u, v \in P, \beta(0)=0, \beta(u) \neq 0$ if $u \neq 0$.

The approach used in proving the existence results in this paper is the following fixed point theorem of cone expansion and compression of functional type due to Avery et al. [34].

Theorem 2.1 Let $\Omega_{1}$ and $\Omega_{2}$ be two bounded open sets in a Banach space E such that $0 \in \Omega_{1}$ and $\Omega_{1} \subseteq \Omega_{2}$ and $P$ is a cone in E. Suppose $T: P \cap\left(\bar{\Omega}_{2} \backslash \Omega_{1}\right) \rightarrow P$ is a completely continuous operator, $\alpha$ and $\gamma$ are nonnegative continuous functional on $P$, and one of the two conditions:

(K1) $\alpha$ satisfies Property $\mathrm{A} 1$ with $\alpha(T u) \geq \alpha(u)$, for all $u \in P \cap \partial \Omega_{1}$, and $\gamma$ satisfies Property A2 with $\gamma(T u) \leq \gamma(u)$, for all $u \in P \cap \partial \Omega_{2}$;

(K2) $\gamma$ satisfies Property A2 with $\gamma(T u) \leq \gamma(u)$, for all $u \in P \cap \partial \Omega_{1}$, and $\alpha$ satisfies Property $\mathrm{A} 1$ with $\alpha(T u) \geq \alpha(u)$, for all $u \in P \cap \partial \Omega_{2}$, is satisfied. Then $T$ has at least one fixed point in $P \cap\left(\bar{\Omega}_{2} \backslash \Omega_{1}\right)$. 


\section{Expression and properties of Green's function}

In this section we present the expression and properties of Green's function associated with FBVP (1.1). We shall consider the Banach space $E=C[0,1]$ equipped with norm $\|u\|=$ $\max _{0 \leq t \leq 1}|u(t)|$. In order to prove our main result, we need some preliminary results.

Lemma 3.1 $([1,2])$ Let $f \in C([0,1] \times[0, \infty)), u \in C[0,1]$, then the boundary value problem

$$
\left\{\begin{array}{l}
{ }^{c} D_{0^{+}}^{\alpha} w(t)+f(t, u(t))=0, \quad 0 \leq t \leq 1, \\
w(0)=w^{\prime}(0)=w^{\prime \prime \prime}(0)=\cdots=w^{(n-1)}(0)=0, \quad w^{\prime \prime}(1)=0,
\end{array}\right.
$$

has a unique solution

$$
w(t)=\int_{0}^{1} G(t, s) f(s, u(s)) d s,
$$

where

$$
G(t, s)=\frac{1}{2 \Gamma(\alpha-2)} t^{2}(1-s)^{\alpha-3}-\frac{1}{\Gamma(\alpha)}[\max \{t-s, 0\}]^{\alpha-1} .
$$

Lemma 3.2 The Green's function $G(t, s)$ defined by (3.2) has the following properties:

(a) $G(t, s) \geq 0, \frac{\partial G(t, s)}{\partial t} \geq 0, \forall t, s \in[0,1]$;

(b) $t^{2} G(1, s) \leq G(t, s) \leq G(1, s), \forall t, s \in[0,1]$;

(c) $\max _{0 \leq t \leq 1} \int_{0}^{1} G(t, s) d s=\int_{0}^{1} G(1, s) d s=\frac{\alpha^{2}-\alpha-2}{2 \Gamma(\alpha+1)}$;

(d) $\min _{\eta \leq t \leq 1} \int_{\eta}^{1} G(t, s) d s=\int_{\eta}^{1} G(\eta, s) d s=\frac{\eta^{2}(1-\eta)^{\alpha-2}}{2 \Gamma(\alpha-1)}$, where $\eta \in(0,1)$;

(e) $\int_{0}^{1} s^{2} G(1, s) d s=\frac{\alpha^{2}+3 \alpha}{\Gamma(\alpha+3)}$.

Proof The properties (a) and (b) one may find in [1]. By direct calculations we obtain (e).

(c) For any $t \in[0,1]$, let $g(t)=\int_{0}^{1} G(t, s) d s$, then

$$
\begin{aligned}
g(t) & =\frac{1}{2 \Gamma(\alpha-2)} \int_{0}^{1} t^{2}(1-s)^{\alpha-3} d s-\frac{1}{\Gamma(\alpha)} \int_{0}^{t}(t-s)^{\alpha-1} d s \\
& =\frac{1}{2 \Gamma(\alpha-1)} t^{2}-\frac{1}{\Gamma(\alpha+1)} t^{\alpha}, \quad t \in[0,1] .
\end{aligned}
$$

It follows from (3.3) that

$$
g^{\prime \prime}(t)=\frac{1}{\Gamma(\alpha-1)}\left(1-t^{\alpha-2}\right) \geq 0, \quad t \in[0,1],
$$

which implies that $g^{\prime}(t)$ is increasing on $[0,1]$, thus

$$
g^{\prime}(t)=\frac{1}{\Gamma(\alpha-1)} t-\frac{1}{\Gamma(\alpha)} t^{\alpha-1} \geq g^{\prime}(0)=0, \quad t \in[0,1],
$$

which implies that $g(t)$ is increasing on $[0,1]$, thus

$$
\max _{0 \leq t \leq 1} \int_{0}^{1} G(t, s) d s=\int_{0}^{1} G(1, s) d s=g(1)=\frac{\alpha^{2}-\alpha-2}{2 \Gamma(\alpha+1)} .
$$


(d) For $t \in[\eta, 1]$, let $h(t)=\int_{\eta}^{1} G(t, s) d s$, then

$$
\begin{aligned}
h(t) & =\frac{1}{2 \Gamma(\alpha-2)} \int_{\eta}^{1} t^{2}(1-s)^{\alpha-3} d s-\frac{1}{\Gamma(\alpha)} \int_{\eta}^{t}(t-s)^{\alpha-1} d s \\
& =\frac{(1-\eta)^{\alpha-2}}{2 \Gamma(\alpha-1)} t^{2}-\frac{1}{\Gamma(\alpha+1)}(t-\eta)^{\alpha}, \quad t \in[\eta, 1] .
\end{aligned}
$$

It follows from (3.4) that

$$
h^{\prime \prime}(t)=\frac{1}{\Gamma(\alpha-1)}\left[(1-\eta)^{\alpha-2}-(t-\eta)^{\alpha-2}\right] \geq 0, \quad t \in[\eta, 1]
$$

which implies $h^{\prime}(t)$ is increasing on $[\eta, 1]$. Thus $h^{\prime}(t) \geq h^{\prime}(\eta)=\eta(1-\eta)^{\alpha-2} / \Gamma(\alpha-1)>0$, $t \in[\eta, 1]$. So, $h(t)$ is increasing on $[\eta, 1]$, therefore,

$$
\min _{\eta \leq t \leq 1} \int_{\eta}^{1} G(t, s) d s=\int_{\eta}^{1} G(\eta, s) d s=h(\eta)=\frac{\eta^{2}(1-\eta)^{\alpha-2}}{2 \Gamma(\alpha-1)} .
$$

Then the proof is completed.

\section{Main results}

In this section, we discuss the existence of positive nondecreasing solution of FBVP (1.1). Define the cone $P$ by

$$
P=\left\{u \in C[0,1]: u(t) \geq 0, u(t) \text { is increasing on }[0,1] \text { and } u(t) \geq t^{2}\|u\|, t \in[0,1]\right\} \text {. }
$$

Then $P$ is a normal cone of $E$. It is easy to see that if $u \in K$, then $\|u\|=u(1)$. Define the operator $T$ by

$$
(T u)(t)=\int_{0}^{1} G(t, s) f(s, u(s)) d s, \quad t \in[0,1]
$$

Then for any $u \in P,(T u)(t)$ is nonnegative and increasing on $[0,1]$ by Lemma 3.2(a). Lemma 3.2(b) implies $(T u)(t) \geq t^{2}\|T u\|, t \in[0,1]$, thus $T(P) \subseteq P$. $T$ is completely continuous by the Ascoli-Arzela theorem. Thus, to solve FBVP (1.1), we only need to find a fixed point of the operator $T$ in $P$. Finally, let us define two continuous functionals $\alpha$ and $\gamma$ on the cone $P$ by

$$
\alpha(u):=\min _{t \in[\eta, 1]} u(t)=u(\eta) \quad \text { and } \quad \gamma(u):=\max _{t \in[0,1]} u(t)=u(1)=\|u\| .
$$

It is clear that $\alpha(u) \leq \gamma(u)$ for all $u \in P$.

Theorem 4.1 Suppose that there exist positive numbers $r, R$ with $r<\eta^{2} R$ such that the following conditions are satisfied:

(C1) $f(t, x) \geq \frac{2 r \Gamma(\alpha-1)}{\eta^{2}(1-\eta)^{\alpha-2}}$ for all $(t, x) \in[\eta, 1] \times[r, R]$,

(C2) $f(t, x) \leq \frac{2 R \Gamma(\alpha+1)}{\alpha^{2}-\alpha-2}$ for all $(t, x) \in[0,1] \times[0, R]$. 
Then FBVP (1.1) has at least one positive and nondecreasing solution $u^{*}$ satisfying

$$
r \leq \min _{t \in[\eta, 1]} u^{*}(t) \quad \text { and } \quad \max _{t \in[0,1]} u^{*}(t) \leq R
$$

Proof Let

$$
\Omega_{1}=\{u: \alpha(u)<r\} \text { and } \Omega_{2}=\{u: \gamma(u)<R\} \text {, }
$$

it is easy to see that $0 \in \Omega_{1}, \Omega_{1}$ and $\Omega_{2}$ are bounded open subsets of $E$. Let $u \in \Omega_{1}$, then we have

$$
r>\alpha(u)=\min _{t \in[\eta, 1]} u(t) \geq \eta^{2}\|u\|=\eta^{2} \gamma(u) .
$$

Thus $R>r / \eta^{2}>\gamma(u)$, i.e., $u \in \Omega_{2}$, so $\Omega_{1} \subseteq \Omega_{2}$.

Claim 1: If $u \in P \cap \partial \Omega_{1}$, then $\alpha(T u) \geq \alpha(u)$.

To see this let $u \in P \cap \partial \Omega_{1}$, then $R=\gamma(u) \geq u(s) \geq \alpha(u)=r, s \in[\eta, 1]$. Thus it follows from (C1), Lemma 3.2(d), and (4.1) that

$$
\begin{aligned}
\alpha(T u) & =(T u)(\eta)=\int_{0}^{1} G(\eta, s) f(s, u(s)) d s \\
& \geq \int_{\eta}^{1} G(\eta, s) f(s, u(s)) d s \geq \frac{2 r \Gamma(\alpha-1)}{\eta^{2}(1-\eta)^{\alpha-2}} \int_{\eta}^{1} G(\eta, s) d s=r=\alpha(u) .
\end{aligned}
$$

Claim 2: If $u \in P \cap \partial \Omega_{2}$, then $\gamma(T u) \leq \gamma(u)$.

To see this let $u \in P \cap \partial \Omega_{2}$, then $u(s) \leq \gamma(u)=R, s \in[0,1]$. Thus condition (C2) and Lemma 3.2(c) yield

$$
\gamma(T u)=(T u)(1)=\int_{0}^{1} G(1, s) f(s, u(s)) d s \leq \frac{2 R \Gamma(\alpha+1)}{\alpha^{2}-\alpha-2} \int_{0}^{1} G(1, s) d s=R=\gamma(u) .
$$

Clearly $\alpha$ satisfies Property A1(c) and $\gamma$ satisfies Property A2(a). Therefore the hypothesis (K1) of Theorem 2.1 is satisfied and hence $T$ has at least one fixed point $u^{*} \in$ $P \cap\left(\bar{\Omega}_{2} \backslash \Omega_{1}\right)$, i.e., FBVP $(1.1)$ has at least one positive and nondecreasing solution $u^{*} \in P$ such that

$$
r \leq \min _{t \in[\eta, 1]} u^{*}(t) \quad \text { and } \quad \max _{t \in[0,1]} u^{*}(t) \leq R
$$

This completes the proof.

Theorem 4.2 Suppose that there exist positive numbers $r, R$ with $r<R$ such that the following conditions are satisfied:

(C3) $f(t, x) \leq \frac{2 r \Gamma(\alpha+1)}{\alpha^{2}-\alpha-2}$, for $(t, x) \in[0,1] \times[0, r]$,

(C4) $f(t, x) \geq \frac{2 R \Gamma(\alpha-1)}{\eta^{2}(1-\eta)^{\alpha-2}}$, for $(t, x) \in[\eta, 1] \times\left[R, R / \eta^{2}\right]$.

Then FBVP (1.1) admits a positive and nondecreasing solution $u^{*} \in P$ such that

$$
r \leq \max _{t \in[0,1]} u^{*}(t) \quad \text { and } \quad \min _{t \in[\eta, 1]} u^{*}(t) \leq R
$$


Proof Let

$$
\Omega_{3}=\{u: \gamma(u)<r\} \text { and } \Omega_{4}=\{u: \alpha(u)<R\} \text {, }
$$

we have $0 \in \Omega_{3}$ and $\Omega_{3} \subseteq \Omega_{4}$, with $\Omega_{3}$ and $\Omega_{4}$ being bounded open subsets of $E$.

Claim 1: If $u \in P \cap \partial \Omega_{3}$, then $\gamma(T u) \leq \gamma(u)$.

To see this let $u \in P \cap \partial \Omega_{3}$, then $u(s) \leq \gamma(u)=r, s \in[0,1]$. Thus condition (C3) and Lemma 3.2(c) yield

$$
\gamma(T u)=(T u)(1)=\int_{0}^{1} G(1, s) f(s, u(s)) d s \leq \frac{2 r \Gamma(\alpha+1)}{\alpha^{2}-\alpha-2} \int_{0}^{1} G(1, s) d s=r=\gamma(u) .
$$

Claim 2: If $u \in P \cap \partial \Omega_{4}$, then $\alpha(T u) \geq \alpha(u)$.

To see this let $u \in P \cap \partial \Omega_{4}$, then $R / \eta^{2}=\alpha(u) / \eta^{2} \geq \gamma(u) \geq u(s) \geq \alpha(u)=R, s \in[\eta, 1]$. Thus it follows from (C4) and Lemma 3.2(d) that one has

$$
\begin{aligned}
\alpha(T u) & =(T u)(\eta)=\int_{0}^{1} G(\eta, s) f(s, u(s)) d s \\
& \geq \int_{\eta}^{1} G(\eta, s) f(s, u(s)) d s \geq \frac{2 R \Gamma(\alpha-1)}{\eta^{2}(1-\eta)^{\alpha-2}} \int_{\eta}^{1} G(\eta, s) d s=R=\alpha(u) .
\end{aligned}
$$

Clearly $\alpha$ satisfies Property A1(c) and $\gamma$ satisfies Property A2(a). Therefore the hypothesis (K2) of Theorem 2.1 is satisfied and hence $T$ has at least one fixed point $u^{*} \in$ $P \cap\left(\bar{\Omega}_{4} \backslash \Omega_{3}\right)$, i.e., FBVP (1.1) has at least one positive and nondecreasing solution $u^{*} \in P$ such that

$$
r \leq \max _{t \in[0,1]} u^{*}(t) \quad \text { and } \quad \min _{t \in[\eta, 1]} u^{*}(t) \leq R
$$

This completes the proof.

Now we discuss nonexistence of positive solutions of FBVP (1.1).

Theorem 4.3 Suppose that $f$ satisfies the condition

$$
\sup _{(t, x) \in[0,1] \times(0, \infty)} \frac{f(t, x)}{x}<\frac{2 \Gamma(\alpha+1)}{\alpha^{2}-\alpha-2} .
$$

Then FBVP (1.1) does not admit positive solutions.

Proof Assume to the contrary that $u=u(t)$ is a positive solution of FBVP (1.1), then

$$
\begin{aligned}
u(1) & =\int_{0}^{1} G(1, s) f(s, u(s)) d s<\frac{2 \Gamma(\alpha+1)}{\alpha^{2}-\alpha-2} \int_{0}^{1} G(1, s) u(s) d s \\
& \leq \frac{2 \Gamma(\alpha+1)}{\alpha^{2}-\alpha-2}\|u\| \int_{0}^{1} G(1, s) d s=u(1) .
\end{aligned}
$$

This is a contradiction and completes the proof. 
Theorem 4.4 Suppose that $f$ satisfies the condition

$$
\inf _{(t, x) \in[0,1] \times(0, \infty)} \frac{f(t, x)}{x}>\frac{\Gamma(\alpha+3)}{\alpha^{2}+3 \alpha} .
$$

Then FBVP (1.1) does not admit positive solutions.

Proof Assume to the contrary that $u=u(t)$ is a positive solution of FBVP (1.1), then

$$
\begin{aligned}
u(1) & =\int_{0}^{1} G(1, s) f(s, u(s)) d s>\frac{\Gamma(\alpha+3)}{\alpha^{2}+3 \alpha} \int_{0}^{1} G(1, s) u(s) d s \\
& \geq \frac{\Gamma(\alpha+3)}{\alpha^{2}+3 \alpha}\|u\| \int_{0}^{1} G(1, s) s^{2} d s=u(1) .
\end{aligned}
$$

This is a contradiction and completes the proof.

\section{Examples}

Now we provide several examples to demonstrate the applications of the theoretical results in the previous sections.

Example 5.1 Consider the fractional boundary value problem

$$
\left\{\begin{array}{l}
{ }^{c} D_{0^{+}}^{7 / 2} u(t)+\frac{t}{3} u^{2}(t)+u(t)+t^{2}+t+1=0, \quad t \in(0,1), \\
u(0)=u^{\prime}(0)=u^{\prime \prime \prime}(0)=u^{\prime \prime}(1)=0 .
\end{array}\right.
$$

In this problem, $\alpha=\frac{7}{2}, f(t, x)=\frac{t}{3} x^{2}+x+t^{2}+t+1$. It is easy to see that $f \in C([0,1] \times$ $[0, \infty),[0, \infty))$. Let $\eta=\frac{1}{2}, r=\frac{1}{24}, R=3$, then $r<\eta^{2} R$,

$$
f(t, x) \leq f(1, R)=9<\frac{35 \sqrt{\pi}}{6}=\frac{2 R \Gamma(\alpha+1)}{\alpha^{2}-\alpha-2}, \quad \text { for }(t, x) \in[0,1] \times[0, R]
$$

and

$$
f(t, x) \geq f(\eta, r)=\frac{6,193}{3,456}>\frac{\sqrt{2 \pi}}{2}=\frac{2 r \Gamma(\alpha-1)}{\eta^{2}(1-\eta)^{\alpha-2}}, \quad \text { for }(t, x) \in[\eta, 1] \times[r, R]
$$

Hence, all of the conditions of Theorem 4.1 are satisfied. Hence, Theorem 4.1 guarantees that FBVP (5.1) has at least one positive solution $u^{*}(t)$ such that

$$
\frac{1}{24} \leq \min _{t \in\left[\frac{1}{2}, 1\right]} u^{*}(t) \quad \text { and } \quad \max _{t \in[0,1]} u^{*}(t) \leq 3
$$

Example 5.2 Consider the fractional boundary value problem

$$
\left\{\begin{array}{l}
{ }^{c} D_{0^{+}}^{9 / 2} u(t)+(2+t) u^{4}(t)+u^{2}(t)+t u(t)+t=0, \quad t \in(0,1) \\
u(0)=u^{\prime}(0)=u^{\prime \prime \prime}(0)=u^{\prime \prime \prime \prime}(0)=u^{\prime \prime}(1)=0
\end{array}\right.
$$


In this problem, $\alpha=\frac{9}{2}, f(t, x)=(2+t) x^{4}+x^{2}+t x+t$. Obviously, $f \in C([0,1] \times[0, \infty),[0, \infty))$. Let $\eta=\frac{1}{2}, R=4, r=1$, then $r<R$,

$$
f(t, x) \leq f(1, r)=6<\frac{189 \sqrt{\pi}}{44}=\frac{2 r \Gamma(\alpha+1)}{\alpha^{2}-\alpha-2}, \quad \text { for }(t, x) \in[0,1] \times[0, r]
$$

and

$$
f(t, x) \geq f(\eta, R)=658.5>240 \sqrt{2 \pi}=\frac{2 R \Gamma(\alpha-1)}{\eta^{2}(1-\eta)^{\alpha-2}}, \quad \text { for }(t, x) \in[\eta, 1] \times\left[R, R / \eta^{2}\right]
$$

Therefore, all the assumptions of Theorem 4.2 hold. Hence, Theorem 4.2 guarantees that FBVP (5.2) has at least one positive solution $u^{*}(t)$ such that

$$
1 \leq \max _{t \in[0,1]} u^{*}(t) \text { and } \min _{t \in\left[\frac{1}{2}, 1\right]} u^{*}(t) \leq 4
$$

Example 5.3 Consider the fractional boundary value problem

$$
\left\{\begin{array}{l}
{ }^{c} D_{0^{+}}^{9 / 2} u(t)+\frac{3 u^{2}(t)+t u(t)}{u(t)+1}(t+\sin t)=0, \quad t \in(0,1) \\
u(0)=u^{\prime}(0)=u^{\prime \prime \prime}(0)=u^{\prime \prime \prime \prime}(0)=u^{\prime \prime}(1)=0
\end{array}\right.
$$

In this problem, $\alpha=\frac{9}{2}, f(t, x)=\frac{3 x^{2}+t x}{x+1}(t+\sin t)$. It is easy to see that $f \in C([0,1] \times$ $[0, \infty),[0, \infty))$. Let $\eta=\frac{1}{2}$, then

$$
\frac{f(t, x)}{x}=\frac{3 x+t}{x+1}(t+\sin t)<6<\frac{189 \sqrt{\pi}}{44}=\frac{2 \Gamma(\alpha+1)}{\alpha^{2}-\alpha-2}, \quad \text { for }(t, x) \in[0,1] \times(0, \infty)
$$

which implies (4.2) holds. Hence, by Theorem 4.3, FBVP (5.3) does not admit positive solutions.

Example 5.4 Consider the fractional boundary value problem

$$
\left\{\begin{array}{l}
{ }^{c} D_{0^{+}}^{7 / 2} u(t)+\frac{7 u^{2}(t)+8 u(t)}{u(t)+1}(2+t+\sin t)=0, \quad t \in(0,1) \\
u(0)=u^{\prime}(0)=u^{\prime \prime \prime}(0)=u^{\prime \prime}(1)=0 .
\end{array}\right.
$$

In this problem, $\alpha=\frac{7}{2}, f(t, x)=\frac{7 x^{2}+8 x}{x+1}(2+t+\sin t)$. It is easy to see that $f \in C([0,1] \times$ $[0, \infty),[0, \infty))$. Let $\eta=\frac{1}{2}$, then

$$
\frac{f(t, x)}{x}=\frac{7 x+8}{x+1}(2+t+\sin t)>14>\frac{1,485 \sqrt{\pi}}{208}=\frac{\Gamma(\alpha+3)}{\alpha^{2}+3 \alpha}, \quad \text { for }(t, x) \in[0,1] \times(0, \infty),
$$

which implies (4.3) holds. Hence, by Theorem 4.4, FBVP (5.4) does not admit positive solutions. 
Authors' contributions

YS conceived of the studies and drafted the manuscript. XZ participated in the discussion. All authors read and approved the final manuscript.

\section{Acknowledgements}

The authors are very grateful to the referees for their valuable suggestions and comments on improving this paper. This work was supported by the Natural Science Foundation of Zhejiang Province of China (LY12A01012).

\section{Received: 1 October 2013 Accepted: 15 January 2014 Published: 31 Jan 2014}

\section{References}

1. Agarwal, RP, Liu, Y, O'Regan, D, Tian, C: Positive solutions of two-point boundary value problems for fractional singular differential equations. Differ. Equ. 48, 619-629 (2012)

2. Tian, C, Liu, Y: Multiple positive solutions for a class of fractional singular boundary value problem. Mem. Differ. Equ. Math. Phys. 56, 115-131 (2012)

3. Ahmad, B, Nieto, JJ: Existence of solutions for nonlocal boundary value problems of higher-order nonlinear fractional differential equations. Abstr. Appl. Anal. 2009, Article ID 494720 (2009)

4. Ahmad, B, Sivasundaram, S: Existence and uniqueness results for nonlinear boundary value problems of fractional differential equations with separated boundary conditions. Commun. Appl. Anal. 13, 121-128 (2009)

5. Babakhani, A, Daftardar-Gejji, V: Existence of positive solutions of nonlinear fractional differential equations. J. Math Anal. Appl. 278, 434-442 (2003)

6. Bai, C: Triple positive solutions for a boundary value problem of nonlinear fractional differential equation. Electron. J. Qual. Theory Differ. Equ. 2010, Article ID 24 (2010)

7. Bai, Z, Qiu, Z: Existence of positive solution for singular fractional differential equation. Appl. Math. Comput. 215, 2761-2767 (2009)

8. Bai, C, Sun, W: Existence and multiplicity of positive solutions for singular fractional boundary value problems. Comput. Math. Appl. 63, 1369-1381 (2012)

9. Bai, C, Sun, W, Zhang, W: Positive solutions for boundary value problems of singular fractional differential equations. Abstr. Appl. Anal. 2013, Article ID 129640 (2013)

10. Caballero, J, Harjani, J, Sadarangant, K: Positive and nondecreasing solutions to a singular boundary value problem for nonlinear fractional differential equations. Commun. Appl. Anal. 15, 265-272 (2011)

11. Caballero Mena, J, Harjani, J, Sadarangani, K: Existence and uniqueness of positive and nondecreasing solutions for a class of singular fractional boundary value problems. Bound. Value Probl. 2009, Article ID 421310 (2009)

12. Ding, $X$, Jiang, $Y$ : Waveform relaxation methods for fractional functional differential equations. Fract. Calc. Appl. Anal. $16,573-594(2013)$

13. El-Shahed, M: Positive solutions for boundary value problem of nonlinear fractional differential equation. Abstr. Appl. Anal. 2007, Article ID 10368 (2007)

14. Jiang, W, Huang, X, Guo, W, Zhang, Q: The existence of positive solutions for the singular fractional differential equation. J. Appl. Math. Comput. 41, 171-182 (2013)

15. Jiang, D, Yuan, C: The positive properties of the Green function for Dirichlet-type boundary value problems of nonlinear fractional differential equations and its application. Nonlinear Anal. 72, 710-719 (2010)

16. Jleli, M, Samet, B: On positive solutions for a class of singular nonlinear fractional differential equations. Bound. Value Probl. 2012, Article ID 73 (2012)

17. Karakostas, GL: Non-existence of solutions for two-point fractional and third-order boundary-value problems. Electron. J. Differ. Equ. 2013, Article ID 152 (2013)

18. Nieto, JJ, Pimentel, J: Positive solutions of a fractional thermostat model. Bound. Value Probl. 2013, Article ID 5 (2013)

19. Qiu, T, Bai, Z: Existence of positive solutions for singular fractional differential equations. Electron. J. Differ. Equ. 2008, Article ID 146 (2008)

20. Wang, Y, Liu, L, Wu, Y: Positive solutions for a class of fractional boundary value problem with changing sign nonlinearity. Nonlinear Anal. 74, 6434-6441 (2011)

21. Xu, X, Jiang, D, Yuan, C: Multiple positive solutions for the boundary value problem of a nonlinear fractional differential equation. Nonlinear Anal. 71, 4676-4688 (2009)

22. Yang, $L, C$ Chen, $H$ : Unique positive solutions for fractional differential equation boundary value problems. Appl. Math. Lett. 23, 1095-1098 (2010)

23. Yuan, C: Multiple positive solutions for $(n-1,1)$-type semipositone conjugate boundary value problems of nonlinear fractional differential equations. Electron. J. Qual. Theory Differ. Equ. 2010, Article ID 36 (2010)

24. Yuan, C, Jiang, D, Xu, X: Singular positone and semipositone boundary value problems of nonlinear fractional differential equations. Math. Probl. Eng. 2009, Article ID 535209 (2009)

25. Zhang, L, Ahmad, B, Wang, G, Agarwal, RP: Nonlinear fractional integro-differential equations on unbounded domains in a Banach space. J. Comput. Appl. Math. 249, 51-56 (2013)

26. Zhang, S: The existence of a positive solution for a nonlinear fractional differential equation. Comput. Math. Appl. 59, 1300-1309 (2010)

27. Zhang, S: Positive solutions to singular boundary value problem for nonlinear fractional differential equation. J. Math. Anal. Appl. 252, 804-812 (2000)

28. Zhang, $X$, Han, Y: Existence and uniqueness of positive solutions for higher order nonlocal fractional differential equations. Appl. Math. Lett. 25, 555-560 (2012)

29. Zhang, X, Liu, L, Wiwatanapataphee, B, Wu, Y: Positive solutions of eigenvalue problems for a class of fractional differential equations with derivatives. Abstr. Appl. Anal. 2012, Article ID 512127 (2012)

30. Zhang, $X, L i u, L, W u, Y$ : The eigenvalue problem for a singular higher order fractional differential equation involving fractional derivatives. Appl. Math. Comput. 218, 8526-8536 (2012)

31. Zhang, X, Liu, L, Wu, Y: Multiple positive solutions of a singular fractional differential equation with negatively perturbed term. Math. Comput. Model. 55, 1263-1274 (2012) 
32. Zhang, $X, L i u, L, W u, Y$ : The uniqueness of positive solution for a singular fractional differential system involving derivatives. Commun. Nonlinear Sci. Numer. Simul. 18, 1400-1409 (2013)

33. Zhang, $X, L i u, L, W u, Y, L u, Y:$ The iterative solutions of nonlinear fractional differential equations. Appl. Math. Comput. 219, 4680-4691 (2013)

34. Avery, R, Henderson, J, O'Regan, D: Functional compression-expansion fixed point theorem. Electron. J. Differ. Equ. 2008, Article ID 22 (2008)

35. Kilbas, AA, Srivastava, HM, Trujillo, JJ: Theory and Applications of Fractional Differential Equations. North-Holland Mathematics Studies, vol. 204. Elsevier, Amsterdam (2006)

36. Podlubny, I: Fractional Diffrential Equations. Math. Sci. Eng., vol. 198. Academic Press, San Diego (1999)

37. Guo, D, Lakshmikantham, V: Nonlinear Problems in Abstract Cones. Academic Press, San Diego (1988)

10.1186/1687-1847-2014-53

Cite this article as: Sun and Zhang: Existence and nonexistence of positive solutions for fractional-order two-point boundary value problems. Advances in Difference Equations 2014, 2014:53

Submit your manuscript to a SpringerOpen ${ }^{\odot}$ journal and benefit from:

- Convenient online submission

- Rigorous peer review

- Immediate publication on acceptance

- Open access: articles freely available online

- High visibility within the field

- Retaining the copyright to your article

Submit your next manuscript at $>$ springeropen.com 